Revue des patrimoines

33 | 2017

Patrimoine culturel immatériel et institutions patrimoniales

\title{
Le numérique comme « lieu » de la sauvegarde du Patrimoine culturel immatériel
}

Digitisation as a 'place' of safeguarding for the intangible cultural heritage

Jean-Jacques Castéret

\section{(2) OpenEdition}

Journals

Édition électronique

URL : http://journals.openedition.org/insitu/15478

DOI : $10.4000 /$ insitu. 15478

ISSN : 1630-7305

Éditeur

Ministère de la culture

Référence électronique

Jean-Jacques Castéret, «Le numérique comme « lieu » de la sauvegarde du Patrimoine culturel immatériel », In Situ [En ligne], 33 | 2017, mis en ligne le 02 novembre 2017, consulté le 19 avril 2019. URL : http://journals.openedition.org/insitu/15478 ; DOI : 10.4000/insitu.15478

Ce document a été généré automatiquement le 19 avril 2019

\section{cc) (1)}

In Situ Revues des patrimoines est mis à disposition selon les termes de la licence Creative Commons Attribution - Pas d'Utilisation Commerciale - Pas de Modification 4.0 International. 


\title{
Le numérique comme « lieu » de la sauvegarde du Patrimoine culturel immatériel
}

Digitisation as a 'place' of safeguarding for the intangible cultural heritage

\author{
Jean-Jacques Castéret
}

1 En France, la diffusion de la notion de Patrimoine culturel immatériel (PCI) a suivi deux voies de façon quasi simultanée. Un mouvement top-down de mise en œuvre, par le ministère de la Culture, de la convention de l'Unesco pour la sauvegarde du PCI (2003) à travers la création, à partir de 2007, d'un inventaire portant sur cette nouvelle catégorie patrimoniale et plus largement, la mobilisation des dispositifs jusqu'alors dédiés au patrimoine ethnologique (appels d'offres, publications, organisation ou aide aux journées d'étude, colloques... $)^{1}$. Envisagée comme un levier par les communautés de praticiens, la convention a également généré un mouvement bottom-up, qu'il s'agisse, dès 2004, de candidatures sur les listes de l'Unesco, d'une participation à l'inventaire et de la diffusion de la notion en régions ${ }^{2}$, cette double dynamique instaurant un dialogue renouvelé entre l'État, les collectivités territoriales, les associations et les acteurs de tous ordres.

2 La transmission des pratiques et savoir-faire représentant l'enjeu majeur pour les communautés et la notion de sauvegarde - donc d'action - étant centrale dans la convention, on peut aujourd'hui s'interroger sur la nature et les cadres de ce dialogue, de même que sur les résultats naissants de ce processus. À la croisée des deux mouvements évoqués ici et à partir de l'expérience conduite par l'ethnopôle InOc Aquitaine, partagée avec le département du Pilotage de la recherche et de la Politique scientifique (DPRPS) du ministère et divers acteurs du PCI du territoire français, cette contribution s'attachera plus particulièrement aux stratégies numériques déployées, et notamment au rôle des dispositifs ainsi produits dans la chaîne patrimoniale ${ }^{3}$. 


\section{Aménagement numérique et $\mathrm{PCI}$}

3 L'InOc Aquitaine est l'opérateur régional pour la langue et la culture occitanes en convention pluriannuelle d'objectifs avec la région, l'État et les départements, et ethnopôle (Pôle national de recherches et de ressources en ethnologie). À partir de 2002, année de création d'une mission de "sauvegarde et transmission du patrimoine oral ", cette catégorie patrimoniale pénètre les schémas publics d'aménagement de l'occitan dans le département des Pyrénées-Atlantiques (2004) et dans la région Aquitaine (2005). Toutefois, la diffusion progressive, en France, de la notion de PCI, et le développement concomitant au sein de l'InOc Aquitaine d'actions de médiation numérique conduisent tout à la fois à la requalification de la mission, à l'augmentation de son périmètre et à la précision de son cadre d'intervention.

4 Dans son cheminement patrimonial et institutionnel, l'InOc Aquitaine bénéficie, en premier lieu, de la dynamique pionnière du programme éditorial «Banque numérique du savoir en Aquitaine » (BnsA), devenu en 2014 Aquitaine Cultures Connectées, mis en œuvre depuis 2000 par la région et la DRAC et inscrit dans les contrats de plan État-région successifs. Les fondations de ce programme, posées en 1998, croisent ${ }^{4}$ en outre celles du plan national de numérisation du patrimoine culturel (1996) portant dans un premier temps sur les fonds iconographiques et sonores appartenant à l'État (réseau des services d'archives, bibliothèques, services de l'Archéologie, Monuments historiques, musées, etc.) puis étendu, à partir de 2000, aux fonds appartenant aux collectivités locales, fondations et associations ${ }^{5}$.

5 En 2003-2005, la mission débutante de l'InOc Aquitaine pour la «sauvegarde du patrimoine oral» est alors en butte à l'absence, dans les territoires aquitains, de dispositifs de financement pour une sauvegarde systématique des fonds d'archives sonores produits par le fort mouvement patrimonial qui s'est tout particulièrement exprimé en Gascogne dans le domaine des musiques et danses traditionnelles et de la littérature orale ${ }^{6}$. Il s'agit de fonds épars, généralement conservés au domicile des collecteurs. Toutefois, à cette époque, la BnsA propose le financement de sites internet pour la valorisation des collections patrimoniales. Le dispositif permet en outre, certes à la marge, la prise en compte de frais de numérisation, de quoi au moins publier un site avec un premier corpus de ressources venant abonder celles, déjà numérisées, dont l'InOc est propriétaire?.

6 À la croisée de la valorisation scientifique et de l'action culturelle, dans une logique de transmission, la création du site internet Sondaqui.com ${ }^{8}$ ne se limite pas à une simple mise en ligne de corpus (fig. 1). Le projet aborde au contraire les archives à partir du vivant. Il prend pour ligne éditoriale «la fête » sous toutes ses formes comme cadre d'expression privilégié de savoir-faire et de pratiques musicales, chorégraphiques, chansonnières, contées... Le site met alors en regard pratiques contemporaines et illustrations sonores puisées dans les fonds archivistiques. Un double mouvement est ainsi produit. Un document d'archives jusqu'alors inaccessible vient illustrer la pratique contemporaine qu'elle nourrit aussi, tout particulièrement au plan stylistique (modalité musicale, rythmique, ornementation, phrasé...). Et le contemporain restitue la densité sociale perdue du document d'archives, l'éclairant par son contexte et rendant aussi parfois plus agréable l'écoute de ces enregistrements anciens collectés auprès de personnes âgées. 
Figure 1

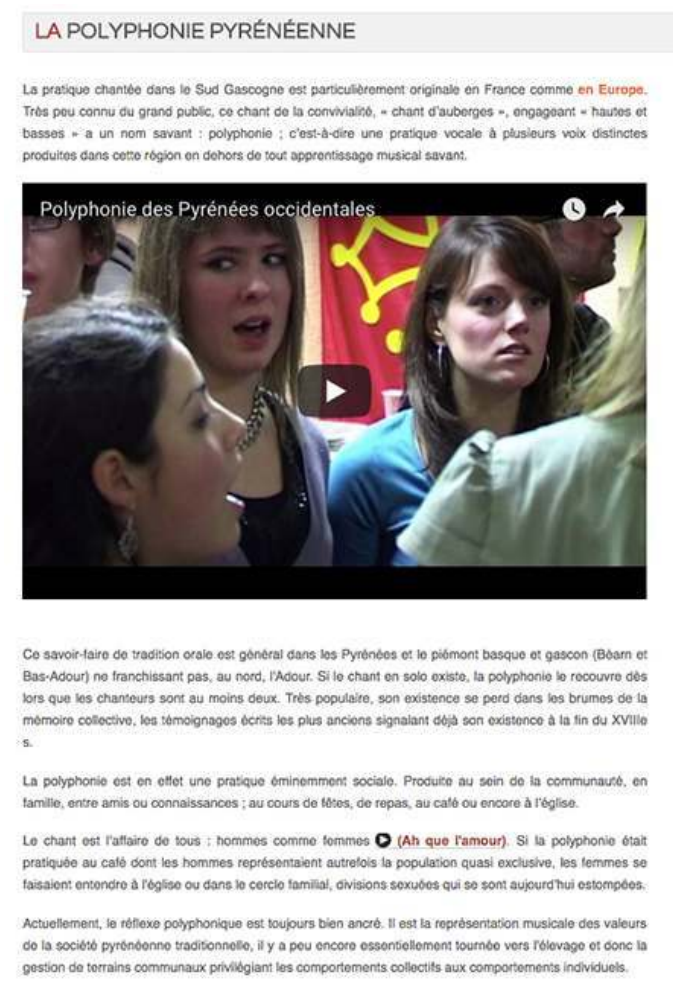

CONSTRUCTION HUMAINE ET MUSICALE

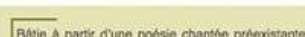
appolso on occtan airo (air), cant (chant) of normala 0 (Dlu d'aqueras montanhetas) (volx "normelon, lo polyphionio ost construbio duno ou d'aqueras montanhetas Cas vox sont improwiblos, Iune dans taigu -

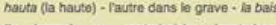
(la basso) ou contrabaisha (controbasso) deux ou trois voix distinctos Les recthiquens vilinthes

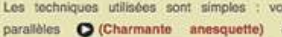
bourdons (notamment un bourdonn bas on 8 ase.

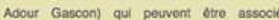
Entencire deux a trois volix ast toutefois tonceiton ou contexto aroxboution : de ta presence do chanteurs aux capachets vocates necossaires capobles dimprovisor hacito of basee, do loum attriess, do meure de rexbecusion, do convivialine dou vin...

Organisation d'une page du site Sondaqui, le site du Patrimoine culturel immatériel occitan en région Aquitaine.

(c) InOc Aquitaine, juillet 2016

7 Le projet, élaboré dans le cadre d'une mission régionale, adopte en outre le périmètre de l'Aquitaine administrative dont la partie occitanophone représente près de $95 \%$ du territoire, conduisant de ce fait à identifier des pratiques vivantes, du moins les plus emblématiques et susceptibles d'être rapprochées des divers fonds de collectes, pour l'ensemble du territoire.

Sondaqui.com, le site du patrimoine oral et festif occitan en Aquitaine, est mis en ligne en décembre 2006. Or, en 2008, au moment où le ministère de la Culture et de la Communication lance son premier appel à projets pour l'inventaire du PCI, la démarche entreprise dans le cadre de la création du site aquitain représente de fait un inventaire dans sa double acception de corpus constitué et de dynamique de terrain, qui ne demande qu'à explorer plus avant les pratiques d'une région cadre. L'InOc Aquitaine participe ainsi de 2008 à 2010 aux premières expériences d'inventaire, sous la maîtrise d'ouvrage du DPRPS du ministère. Cet «Inventaire pilote du Patrimoine culturel immatériel (PCI) d'expression occitane en région Aquitaine $"^{9}$ est suivi d'autres opérations d'inventaire, thématiques cette fois, portées par le laboratoire ITEM (EA 3002) de l'université de Pau et des Pays de l'Adour, en collaboration avec l'InOc Aquitaine qui intervient plus particulièrement sur le volet de la valorisation numérique.

9 En effet, à partir de 2012, les partenaires publics s'accordent pour faire de Sondaqui l'outil régional de valorisation numérique. Or, au bénéfice de sa réactualisation technologique et, suite aux travaux d'inventaire, sa ligne éditoriale est étendue au patrimoine culturel 
immatériel. Dans le paysage français émergent en matière de PCI, un dispositif public régional de sauvegarde - au sens de la convention de 2003 - naît de ce fait (fig. 2).

Figure 2

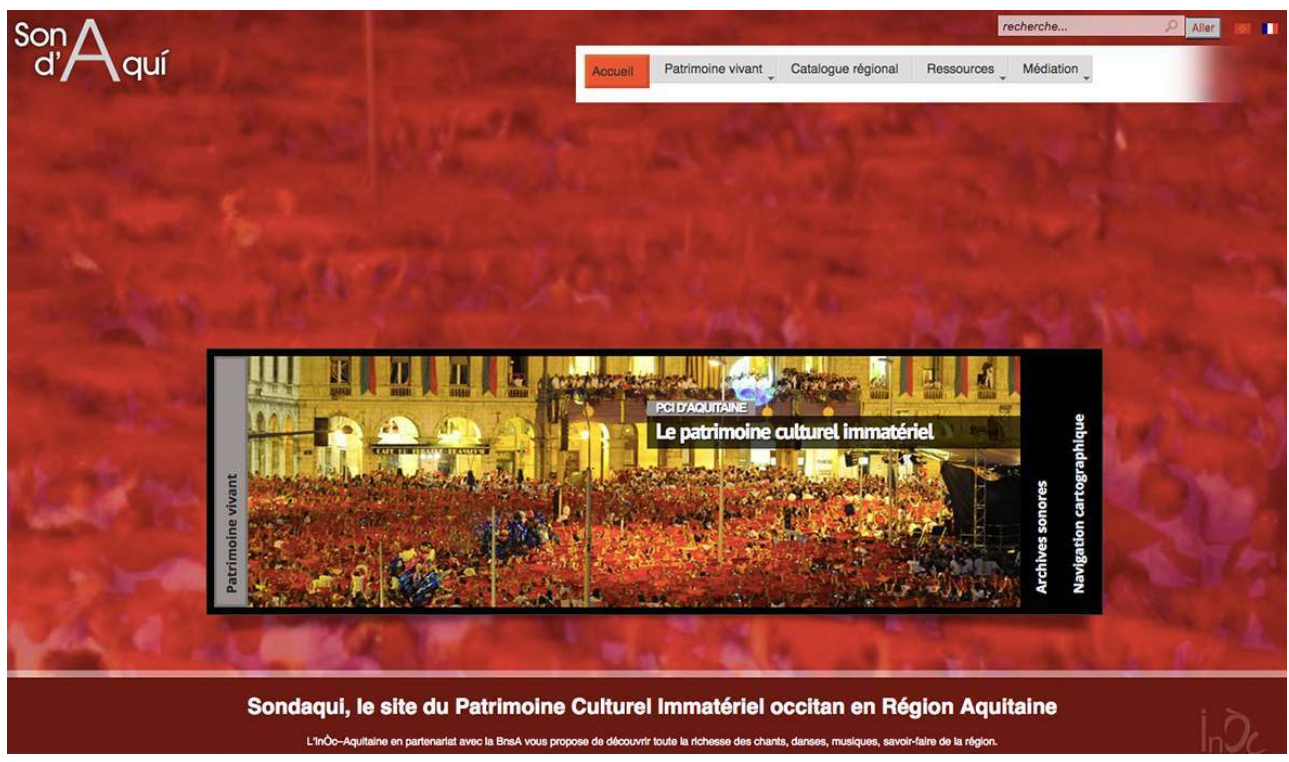

Page d'accueil de Sondaqui, le site du Patrimoine culturel immatériel occitan en région Aquitaine.

(c) InOc Aquitaine, juillet 2016.

En 2014, la création du site JocondeLab ${ }^{10}$, sous l'égide de la Délégation générale à la langue française et aux langues de France ${ }^{11}$, site expérimental explorant les possibilités du " web sémantique ou 'Web 3.0' et de la mise en relation de données culturelles 'liées', tant en termes de multilinguisme, que d'ergonomie et d'interactivité " laisse entrevoir tout l'intérêt de ce type d'approche pour le domaine du PCI. Si cette expérimentation cherche à faciliter, auprès de nouveaux publics, l'accès aux quelques 300000 œuvres des collections de la réunion des musées de France par une approche multilingue incluant les langues de France, elle explore en outre de nouvelles formes de navigation de même que le principe d'une indexation collaborative à partir des données issues de l'encyclopédie en ligne Wikipédia.

11 Après huit années de collaboration avec le DPRPS et dans la dynamique des échanges réguliers que ce dernier organise avec les divers acteurs français du domaine, formant de fait une " communauté », l'InOc Aquitaine propose ainsi la création d'un site collaboratif pour la valorisation publique de l'inventaire reposant sur le liage de données, soit une application de deuxième génération - après JocondeLab -, tel que le souhaitait du reste, dès l'origine, le ministère. Dans un contexte de diversité culturelle et linguistique, PciLab ${ }^{12}$ est ainsi une exploration tant technologique que méthodologique, humaine et partenariale à l'échelle nationale pour l'inventaire du PCI en France ${ }^{13}$.

\section{Écritures numériques}

12 Les projets d'écriture correspondant aux réalisations successives, plus particulièrement sous-tendus par la question de la description du PCI, ont motivé l'exploration des possibilités offertes par les évolutions technologiques dans le domaine numérique. La 
notion de PCI recouvre en effet des savoir-faire et pratiques rarement isolés mais au contraire, la plupart du temps, associés dans des systèmes par essence complexes, qui recouvrent les champs du symbolique, de l'économique, du social, du rituel, de l'artistique ${ }^{14}$. «La promenade des bœufs gras en Bazadais » (sud-Gironde) constitue par exemple un système de rituels au temps du carnaval mêlant problématiques agroalimentaires, festives et musicales. Engraissés avec soin et magnifiquement parés, les bœufs sont promenés jusqu'à l'abattoir, l'ensemble du rituel étant accompagné par un orchestre traditionnel. De la même façon, foires, pèlerinages, fontaines de guérison et même jeux sont généralement combinés. La "foire de la Saint-Michel à Ousse-Suzan " (Landes), le 29 septembre, associe lieux de culte (trois fontaines miraculeuses), une chapelle du XII ${ }^{e}$ s. et une grande foire-braderie agricole, à la croisée et tout le long de deux routes, attirant plus de 500 exposants et plusieurs dizaines de milliers de visiteurs. De même, à Lavaur (Dordogne), le rassemblement annuel à la " fontaine des trois évêques " relève d'une rencontre des patrimoines naturel, lithique, légendaire, du rituel païen et de l'expression religieuse catholique. Or, toutes ces imbrications de patrimoines font anthropologiquement sens dès lors que l'on peut les aborder de façon systémique. Dans un projet éditorial, comment, ainsi, restituer, décrire, faire éprouver à l'internaute ces systèmes?

13 Dans ses deux premières versions, le site Sondaqui.com présente le $\mathrm{PCI}$ à l'aide des fonctionnalités permises par le Web 1.0. Dès la conception du site, soit dès la définition de son architecture, les possibilités de navigation par liens hypertexte ont été au cœur du projet éditorial, exploitées au maximum des occasions de sérendipité ${ }^{15}$. Par ailleurs, dans cet environnement, la description du PCI procède d'une architecture arborescente, du général au particulier. Ainsi, le «Patrimoine vivant $»^{16}$, catégorie la plus large du site, accessible depuis le menu principal et l'accueil, permet d'accéder à diverses souscatégories - Fêtes, Arts et Artisanat - donnant elles-mêmes accès à d'autres rubriques ou à des articles qui les composent. Les fêtes sont par exemple présentées selon une typologie distinguant "fêtes patronales", «calendaires" ou même thématiques ("métiers", "festivals»). L'immersion de l'internaute dans le terrain des pratiques vivantes prend alors la forme d'une exploration conduite et structurée, prescrite, par le chercheur. L'internaute devient toutefois acteur de sa visite dès lors qu'il atteint l'article, c'est-à-dire la page internet présentant une pratique: "Les fêtes de Laruns", le «carnaval de Géronce »... Chaque page est en effet reliée à d'autres par les hyperliens - rendus possibles grâce au langage HTML - qui permettent, dans une certaine mesure, de rendre compte de la porosité des catégories et de l'imbrication des expressions culturelles. Dans le cas de la page «Fêtes de Laruns $»^{17}$ où s'expriment abondamment pratiques vocales polyphoniques, danse du branle au son du couple instrumental flûte à trois trous/tambourin à cordes, chaque mention de ces pratiques et savoir-faire permet un accès à la page descriptive correspondante illustrée de nombreux documents sonores et audiovisuels. Il en va de même pour chaque page atteinte, reliée à toutes les pages ayant un dénominateur commun.

Dans un contexte "Web 1.0 », la valorisation numérique en ligne, pour peu qu'elle organise une navigation de relation en relation, est ainsi particulièrement adaptée à faire expérimenter la richesse des systèmes de pratiques. Pour autant, tous les articles n'étant pas reliés entre eux, la sérendipité reste limitée et ce d'autant plus que chaque article ne peut être consulté que depuis une catégorie générale, accessible depuis la page d'accueil. À l'inverse, une approche sémantique rompt avec une navigation linéaire, comme si l'on 
feuilletait un livre, engageant l'internaute à composer lui-même sa navigation. En effet, cette approche laisse l'internaute libre de naviguer du particulier - c'est-à-dire de la pratique, de l'objet - vers le général - comme les catégories de pratiques - et inversement. Ainsi, lorsqu'il accède à la page d'accueil de JocondeLab, douze notices descriptives d'œuvres d'art des collections des musées de France sont affichées de façon aléatoire. Celles-ci sont décrites par des tags sémantiques sur lesquels l'internaute clique afin de lancer une recherche qui lui permettra d'accéder à l'ensemble des œuvres ayant une relation thématique avec l'œuvre de départ.

Ce type de navigation a donc servi de postulat de départ pour la création de PciLab, prenant le particulier comme point d'entrée de la navigation et permettant à l'internaute, à partir de là, de découvrir le système complexe dans lequel elle s'inscrit.

Le projet a toutefois introduit une variante afin de guider le grand public confronté tout à la fois à un océan de pratiques et à une catégorie patrimoniale encore méconnue. Entre le particulier et le trop général, et afin de favoriser la sérendipité, ce site privilégie donc comme "point d'immersion » l'un des champs descripteurs de la notice d'inventaire, le champ « domaine ». Celui-ci renvoie à des thématiques compréhensibles par le grand public (jeux, art du conte, pratiques rituelles, pratiques festives, savoir-faire, musiques et danses, pratiques sportives $\left.{ }^{18}\right)$ mais sans valeur absolue au regard du PCI. En effet, bien des pratiques (et donc des fiches d'inventaire) peuvent participer de plusieurs catégories différentes : la «Promenade des bœufs gras » de Bazas relève tout autant du rituel, du festif, du musical que des savoir-faire agropastoraux. L'approche sémantique permet toutefois de respecter les chevauchements de domaines, de restituer la porosité des classifications grâce aux concepts descripteurs (les tags) multipliables à l'envi dans chacun des champs de la notice d'inventaire. Elle permet, en outre, une souplesse et une économie d'écriture au regard du Web 1.0 ou 2.0, en évitant de recourir à des scénarios et des navigations - par trop complexes pour rendre compte de vastes champs culturels (fig. 3). 


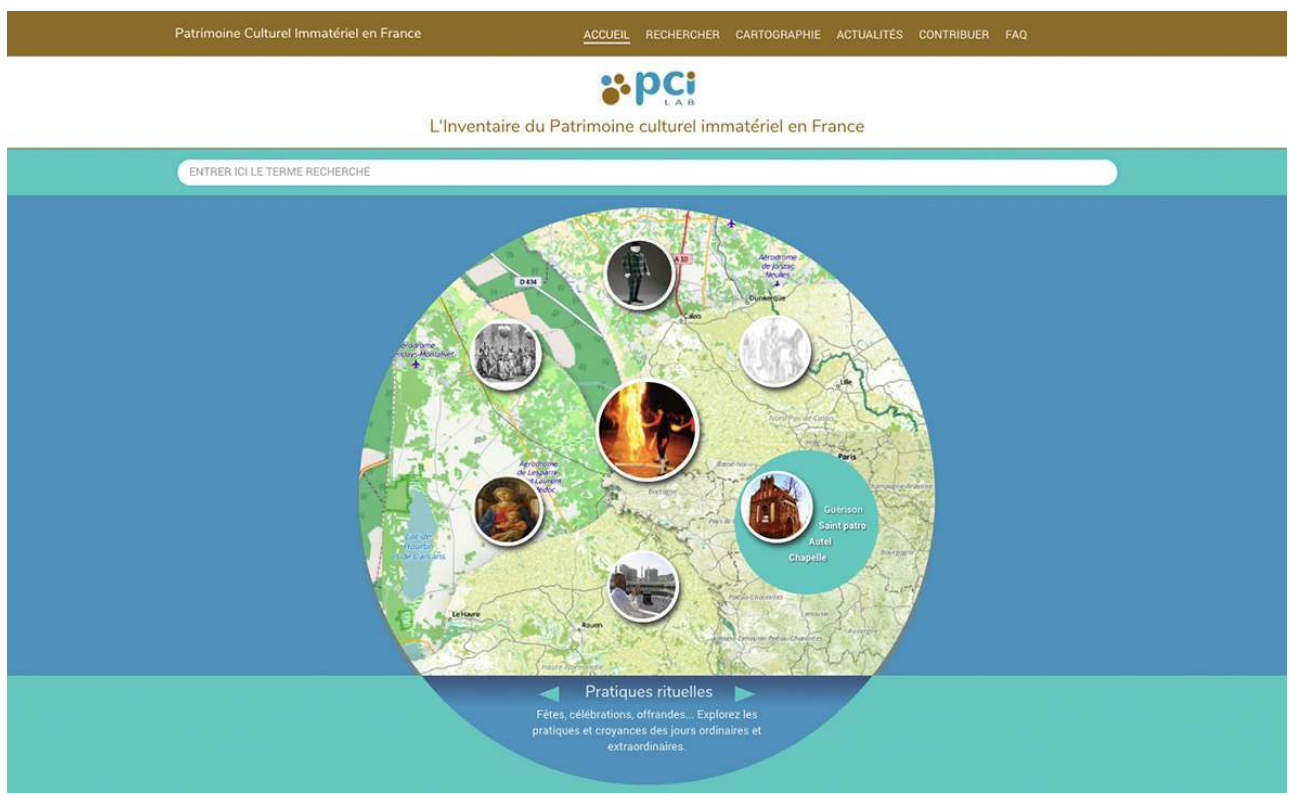

L'un des écrans aléatoires d'accueil de PciLab, le site de l'inventaire français du patrimoine culturel immatériel.

(c) InOc Aquitaine, juillet 2016. (c) InOc Aquitaine - DPRPS du ministère de la Culture, octobre 2017.

\section{Médiation et stimulus numérique}

L'enjeu de la création d'outils numériques dépasse cependant la question de la transcription éditoriale du PCI à l'adresse des publics. En effet, si les outils présentés sont des fenêtres ouvertes sur les expressions et savoir-faire vivants, ils sont non seulement des leviers permettant de stimuler cette vitalité mais encore des espaces de vie.

En dehors de l'intérêt intrinsèque - thématique - d'un site internet, la fréquentation de celui-ci dépend autant de la richesse de ses contenus, de leur enrichissement périodique que des animations organisées autour d'eux. L'InOc Aquitaine a ainsi, depuis 2013, mis en place un protocole d'animation, tout d'abord en communiquant sur les contenus de Sondaqui grâce à ses lettres électroniques « clinhets » puis, à partir de 2015, directement à travers les réseaux sociaux, la page Facebook de l'InOc Aquitaine affiche aussi, au même titre que d'autres expressions culturelles et linguistiques du domaine occitan, l'actualité des pratiques du PCI. Chaque semaine ou au gré du calendrier, le bandeau d'accueil de la page affiche ainsi une pratique ou un événement nouveau. De nombreuses informations concernant le territoire aquitain ou l'actualité du PCI en France sont également postées chaque semaine. Des quizz viennent en outre stimuler les internautes.

Un double mouvement en découle. En premier lieu, les publications ciblées proposant l'adresse de la page du site internet Sondaqui, correspondant à la pratique, conduisent ainsi des internautes à consulter cette page. Certaines pratiques culturelles ou périodes de l'année, comme les premiers jours des congés de Noël, peuvent aussi donner lieu à des pics de fréquentation - 500 connexions au site en un seul jour pour la page consacrée aux "Halhas de Nadau " ${ }^{19}$ (feux de Noël) -. Chaque action sur le réseau social - mention «J'aime» (Facebook), commentaire, partage - affirme et réaffirme par ailleurs 
l'attachement de l'internaute à une communauté de pratiques qui se renforce d'autant. Elle étend aussi tout à la fois l'audience du site ressource, et de façon plus appréciable encore, celle de la manifestation elle-même qui voit son prestige - son capital symbolique - s'accroître. Des flux en résultent. «Partages » et « personnes atteintes » (c'est-à-dire le nombre de personnes uniques ayant vula publication, l'internaute ne verrait-il qu'un titre ou une photographie) mettent également en évidence sur les réseaux une dynamique qui, si elle n'est pas la vie même de la pratique, affiche toutefois une forme de vitalité dont elle devient une part constitutive. Ce mouvement plus ou moins important à l'échelle d'un seul message (mais plusieurs informations par jour peuvent concerner le PCI, d'un bal en Périgord à un carnaval de renom en passant par un séminaire ou un colloque) contribue encore à donner corps à la notion de PCI.

Dans le contexte présent, établir un lien entre un site de contenus à vocation patrimoniale et une animation via les réseaux sociaux forme un dispositif de stimulation de la communauté (communauté de porteurs d'expressions du PCI-internautes et d'internautes potentiellement captifs dans ces domaines). Il permet la stimulation d'un " désir de PCI » sans lequel une pratique ne peut se perpétuer (fig. 4).

Figure 4

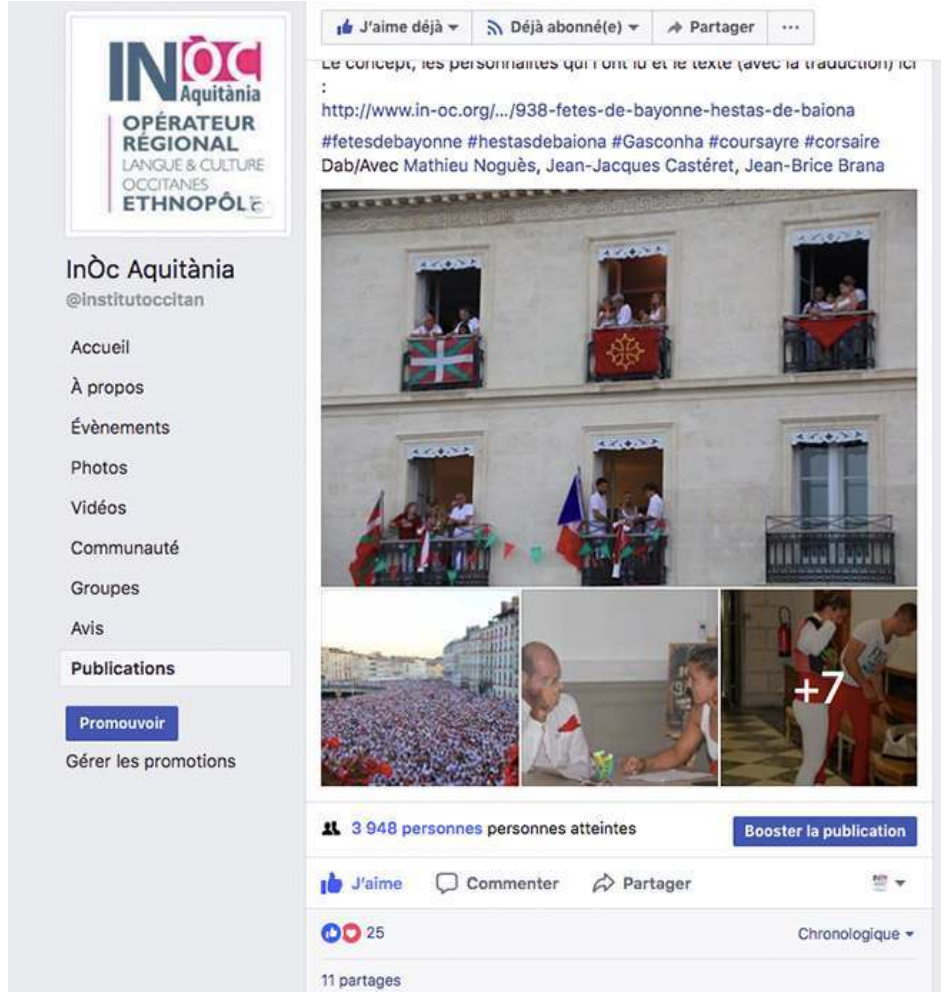

Publication sur la page Facebook de l'InOc Aquitaine à l'occasion de l'ouverture des Fêtes de Bayonne. (c) Facebook / InOc Aquitaine, juillet 2017.

\section{Centre de ressources}

21 L'outil numérique est aujourd'hui un centre de ressources en ligne donnant un accès direct à des textes, des archives manuscrites, des documents audio et audiovisuels. Il permet en outre de réaliser des ensembles thématiques adaptés à des publics spécifiques. 
C'est le cas de Sondaqui dont la dimension musicale première a notamment, dès sa mise en ligne, attiré un public spécialisé : enseignants et formateurs, étudiants et, plus largement, musiciens. Cette plateforme documentaire ${ }^{20}$ permet en effet de croiser ressources publiques, associatives ou privées, donnant accès à des archives sonores jusqu'alors inaccessibles, que ce soit en raison des restrictions à la consultation émises par les chercheurs institutionnels dans le cas du MuCEM (ancien MNATP-Musée national des arts et traditions populaires) ${ }^{21}$, ou en l'absence, en Aquitaine, dans les années 1990, d'organisation associative ou publique en capacité de prendre en charge le récolement et la numérisation des enregistrements produits par les collecteurs ayant œuvré dans les années 1970 et 1980. Avec la création de Sondaqui, des formateurs ou étudiants en musiques et danses traditionnelles, notamment du pôle d'enseignement supérieur Musique et Danse Bordeaux-Aquitaine (ancien Cefedem Aquitaine-Centre de formation des enseignants de la danse et de la musique), du CESMD-centre d'études supérieures musique et danse Poitou-Charentes et des conservatoires régionaux de Limoges et de Toulouse, consultent et utilisent régulièrement les archives en ligne du site pour préparer leurs cours ou mémoires et nourrir leur pratique, sollicitant également un accès plus large à des fonds non publiés.

Outils numériques et professionnels de l'InOc Aquitaine sont également mobilisés par des artistes s'exprimant dans le champ des musiques traditionnelles ou provenant d'autres esthétiques participant des musiques actuelles. Sondaqui sert ainsi de ressource et de support dans le cadre de la «Résidence de création d'essence patrimoniale Va sampler ta grand-mère » portée depuis 2012 par le groupe Familha Artús et le collectif Ça-i en partenariat avec l'InOc Aquitaine et l'association AMPLI (Agglomération Pau-BéarnPyrénées) qui accueille la résidence. Il s'agit d'un projet d'accompagnement et de création musicale sur le thème de la culture occitane en lien avec les musiques actuelles. Celui-ci s'adresse à de jeunes groupes amateurs (jusqu'alors des formations électro, métal, reggae et slam) du département des Pyrénées-Atlantiques afin de leur permettre de s'approprier le patrimoine occitan. L'enjeu est ici de transmettre cette matière afin qu'elle soit utilisée comme source de création. Va sampler... invite donc les jeunes musiciens à s'intéresser aux expressions du PCI dans leur entourage immédiat en réalisant une enquête sonore. Une présentation des contextes sociaux, des formes musicales, des systèmes de pratiques est également organisée, au château d'Este, à Billère, siège de l'InOc Aquitaine, prolongée par une incitation à la navigation sur Sondaqui tout au long de la résidence. Le processus de création s'appuie donc autant sur ces éléments de présentation que sur les matériaux collectés (écrits/oraux, directs/indirects) issus du patrimoine culturel immatériel du territoire concerné. Les artistes vont alors les analyser, les utiliser et les transcender grâce à la rencontre avec des musiciens professionnels du groupe Artús avec lesquels une restitution en concert est organisée, par exemple dans le cadre du très populaire «Hestiv'Oc », festival des Cultures et Musiques du Sud, organisé à Pau depuis 2005.

Avec l'affirmation croissante, depuis 2013, de l'éducation artistique et culturelle (EAC), et notamment son inscription dans la loi sur l'éducation et la circulaire commune Éducation nationale/Culture ${ }^{22}$, une mission visant la structuration de parcours d'EAC pour le domaine occitan a été inscrite dans la convention InOc Aquitaine 2015-2017 proposée par la DRAC et la région. Sa mise en œuvre associe définition et organisation de parcours dans les établissements d'enseignement, et accès aux ressources numériques.

Sondaqui a ainsi servi de support à l'Itinéraire de découvertes «chant et danse de Gascogne» du collège Joseph-Peyré de Garlin (Pyrénées-Atlantiques), proposé en 
2015-2016 aux élèves de quatrième de cet établissement. Il s'agit pour les enseignants référents du collège d'aborder la transmission en s'appuyant successivement sur de classiques ateliers de danse, animés par des intervenants extérieurs spécialistes du domaine qui procèdent par explication et imitation, puis sur les ressources numériques mobilisables par l'InOc Aquitaine permettant d'enrichir le programme avec une mise en perspective patrimoniale: musicale, anthropologique, chorégraphique, musicologique, linguistique et muséographique. Celle-ci inclut des recherches sur la scène artistique occitane contemporaine et des séances d'élargissement à des pratiques similaires en Europe.

Les élèves sont en outre tour à tour récepteurs puis transmetteurs car, si le projet prend sa source dans le cadre scolaire, il s'ouvre sur l'ensemble de la communauté sous la forme d'un bal gascon, organisé en fin d'année avec le collectif Ça-i, la communauté de communes, la commune et le comité des fêtes. Là, les collégiens interviennent à la fois comme praticiens de la transmission dans un atelier de danse d'une heure, conçu par leurs soins, au cours duquel ils transmettent leur savoir aux adultes et aux autres adolescents; puis, lors de la soirée du PCI, en assurant la première partie du bal, la seconde étant mise en œuvre par le groupe Mossur, la formation de bal du groupe Artús.

L'outil numérique dédié au PCI s'inscrit ainsi dans une chaîne sociétale alliant communauté éducative, comité des fêtes et collectivité. En outre, les séquences filmées de transmission réalisées par les adolescents étant produites sous forme de tutoriels ${ }^{23}$, euxmêmes postés sur les réseaux sociaux, elles agissent ainsi, en complément de Sondaqui, tout autant comme sources - médias de la transmission - que comme réceptacles supports d'un nouveau cycle patrimonial ${ }^{24}$.

\section{Numérique et trans-mission}

Parallèlement à cette expérience, afin de mettre à disposition des classes (enseignants et élèves) un portail dédié, Daquidoc.org ${ }^{25}$, «module web langue et culture occitane pour l'EAC », a été produit dans le cadre du dispositif Aquitaine Cultures Connectées (fig. 5). Il permet un accès privilégié aux ressources numériques utiles aux parcours d'EAC, qu'elles soient produites par l'InOc Aquitaine (Sondaqui.com, Troubadours d'Aquitaine ${ }^{26}$, in-oc.org ${ }^{27}$, l'exposition numérique Extraordinaire quotidien ${ }^{28}$ ) ou par d'autres organismes publics ou privés : CAP'Òc (Centre d'animation pédagogique en occitan du réseau Canopé), Cirdoc (Centre interrégional de développement de l'occitan), associations de musiques et danses traditionnelles... Les données identifiées et référencées par l'InOc sont alors consultables par niveau (cycles éducatifs), par thématique, de façon chronologique ou géographique. 


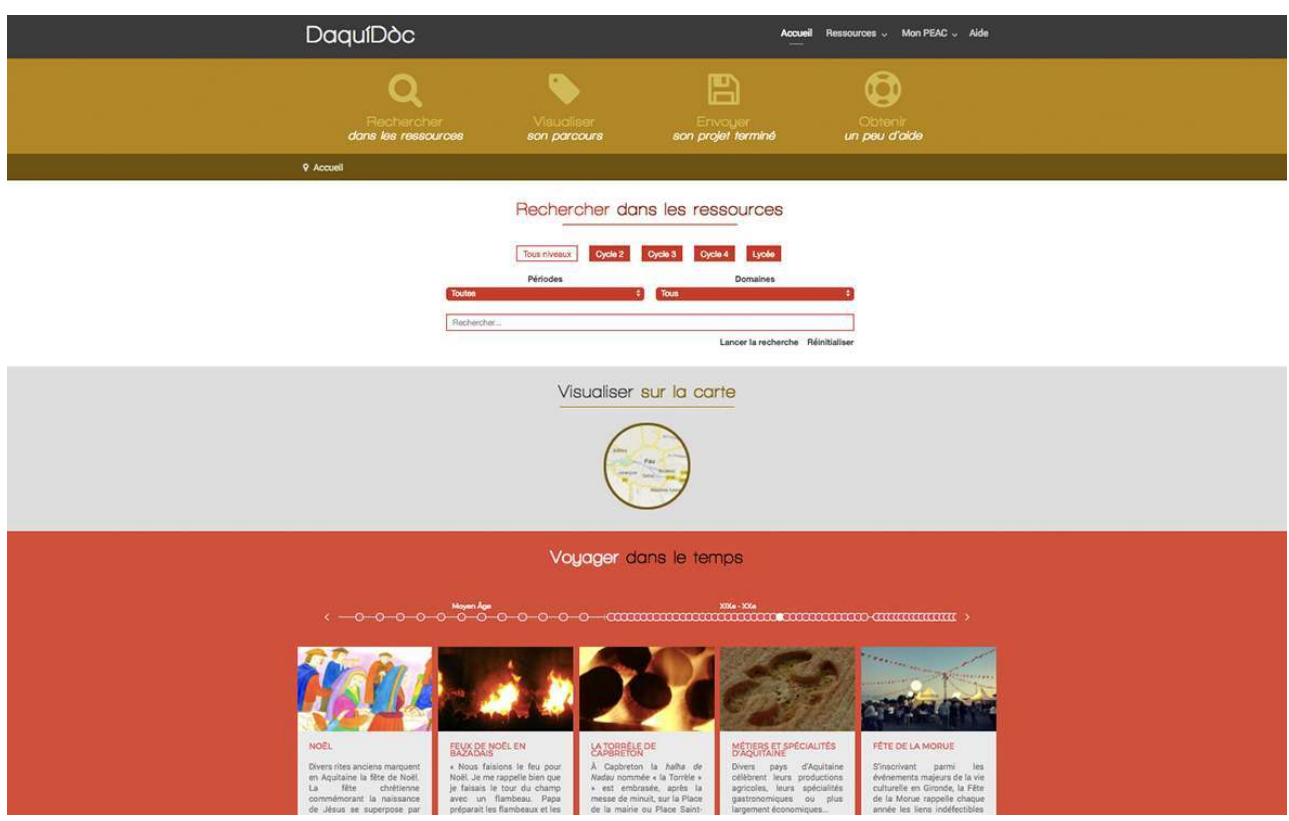

Écran de recherche de DaquiDoc, module web langue et culture occitane pour l'EAC.

(c) InOc Aquitaine, janvier 2016.

L'utilisation du module fait l'objet, en 2016-2017, d'une expérimentation dans le cadre de parcours bâtis par des enseignants volontaires et par l'InOc, en qualité d'opérateur culturel. Celui-ci accompagne l'enseignant dans la création des contenus artistiques ou culturels, ainsi que la classe, en guidant les élèves dans le choix et la conduite d'exposés, en présentant son expérience professionnelle de même que les pratiques et savoir-faire, notamment à partir des ressources numériques de DaquiDoc.

La découverte du patrimoine culturel immatériel est ainsi abordée selon des approches différentes selon les classes. Il est par exemple considéré en tant que catégorie patrimoniale avec une classe de seconde du lycée Louis-Barthou de Pau (PyrénéesAtlantiques), dans le cadre d'un enseignement d'exploration «création et activités artistiques, option Patrimoines». Ce «Parcours de découverte du patrimoine culturel immatériel occitan en Nouvelle-Aquitaine ", composé de six séances, permet ainsi d'une part d'explorer la notion de "patrimoine », et d'autre part, de découvrir la variété des formes du PCI en se basant sur l'environnement culturel immédiat de cet établissement de la capitale béarnaise. Le parcours aborde les notions de classification et d'inventaire, tant à l'échelle nationale que régionale, en amenant les élèves à s'interroger sur les conditions qui font qu'une pratique peut devenir patrimoniale. Il présente les filières universitaires et les métiers qui étudient, valorisent, gèrent, développent le $\mathrm{PCI}$ via des rencontres avec un professionnel de l'InOc Aquitaine.

Dans le cas d'une classe pour l'inclusion scolaire de Podensac (Gironde), scolarisant des enfants de cycle élémentaire en situation de handicap, le parcours est cette fois centré sur une pratique : le jeu de quilles de $\operatorname{six}^{29}$, bien vivant en Gascogne et récemment documenté à la croisée des travaux d'inventaire occitano-aquitain (InOc Aquitaine/laboratoire ITEM de l'université de Pau et des Pays de l'Adour ${ }^{30}$ ), et de l'inventaire des jeux traditionnels en France $^{31}$ (laboratoire IDEMEC de l'université d'Aix-Marseille). Dans ce cadre, aux aspects historiques et ethnographiques sont associés l'apprentissage des règles et du lexique 
occitan spécialisé, la découverte des éléments matériels du jeu et de leur artisanat. Enfin, la pratique elle-même, notamment à travers l'organisation de rencontres interclasses ou inter-écoles.

\section{Espaces et instances collaboratifs}

31 La plateforme de ressources documentaires créée dans le cadre de Sondaqui constitue une première approche collaborative qui rassemble collecteurs et institutions. Cette logique trouve une nouvelle transcription dans l'environnement du Web 3.0. PciLab apporte en effet une perspective collaborative à l'Inventaire. En premier lieu, sa création - et déjà les échanges ayant présidé à celle-ci dans une logique de co-construction des fonctionnalités de l'outil - équivalent à la mise en place d'un organe propre à la communauté des acteurs de l'inventaire. Il constitue un agrégateur d'énergies et d'ambitions partagées pour le PCI qui ont notamment, lors de la réunion annuelle des contributeurs à l'inventaire français, organisée en juillet 2016 à la MMSH d'Aix-en-Provence, présidé à une évolution de son périmètre. L'outil n'est plus depuis seulement dédié à l'accès à l'Inventaire français (c'està-dire aux fiches d'inventaire) mais il est déployé en un portail affichant l'actualité du PCI en France, à même de valoriser les expériences de médiation conduites dans les régions, à l'instar du projet de bande dessinée porté par le service culturel du centre hospitalier universitaire de Rouen à la suite des travaux d'inventaire de Yann Leborgne sur le PCI du milieu hospitalier; les travaux d'inventaire participatif conduits, en Bretagne, par l'association Bretagne Culture Diversité ; l'enquête dirigée par Francesca Cominelli (IREST université Paris-1-Panthéon-Sorbonne) sur les bonnes pratiques de sauvegarde du PCI en France. Le portail articule ainsi les dimensions régionales et nationale $\mathrm{du} \mathrm{PCI}$.

PciLab permet en outre un second niveau de collaborativité de par son lien organique avec Wikidata ${ }^{32}$, la base de connaissances de Wikipédia. En effet, si PciLab ne permet pas aux internautes de modifier en ligne les fiches d'inventaire puisque celles-ci - même si elles peuvent évoluer dans le temps - ont fait l'objet d'un protocole de validation et d'inscription par le ministère, le lien entretenu avec Wikipédia facilite en revanche l'amorce d'un « inventaire libre ", chaque fiche de l'Inventaire français ayant son pendant dans Wikipédia, qu'il existe à l'état embryonnaire ou qu'il soit déjà très développé.

Définition du PCI et philosophie de l'encyclopédie libre entretiennent d'ailleurs des affinités, à commencer par le rôle dévolu à la «communauté ». Un dialogue a ainsi pu s'engager à l'occasion de journées organisées au Centre français du PCI à Vitré (Ille-etVilaine) réunissant Wikimédia France, l'InOc Aquitaine et le DPRPS du ministère. Dans ce cadre, l'identification sur Wikipédia d'une dynamique PCI a grandement facilité le travail de l'équipe en charge de la création de PciLab. Ces journées ont aussi conduit Mélanie Larché, chargée de la coordination de ce dossier au sein de l'InOc dans le cadre d'une thèse de doctorat en contrat Cifre, à revisiter l'encyclopédie libre en mettant à jour diverses références afférentes au PCI, actualisant par exemple la notion ancienne de «trésors humains ». Un tel travail sur la version francophone de l'encyclopédie a dès lors entraîné l'harmonisation d'autres versions : arabe, flamande...

De nouvelles perspectives s'ouvrent aujourd'hui afin de stimuler la contribution à l'Inventaire sur Wikipédia par le biais d'éditathons, c'est-à-dire des journées thématiques contributives permettant de faire se rencontrer les deux communautés: amener les wikipédiens actifs à découvrir et documenter le PCI, que ce soit en français ou dans les diverses langues de France et, de la même façon, permettre aux porteurs d'expressions et 
de savoir-faire du PCI d'en témoigner sur Wikipédia (fig. 6). Celui-ci pourrait ainsi constituer dans l'avenir un sas pour l'Inventaire français: une nouvelle forme d'inventaire libre collaboratif qui serait aussi pour les chercheurs et pour le ministère un lieu de repérage de pratiques ignorées.

Figure 6

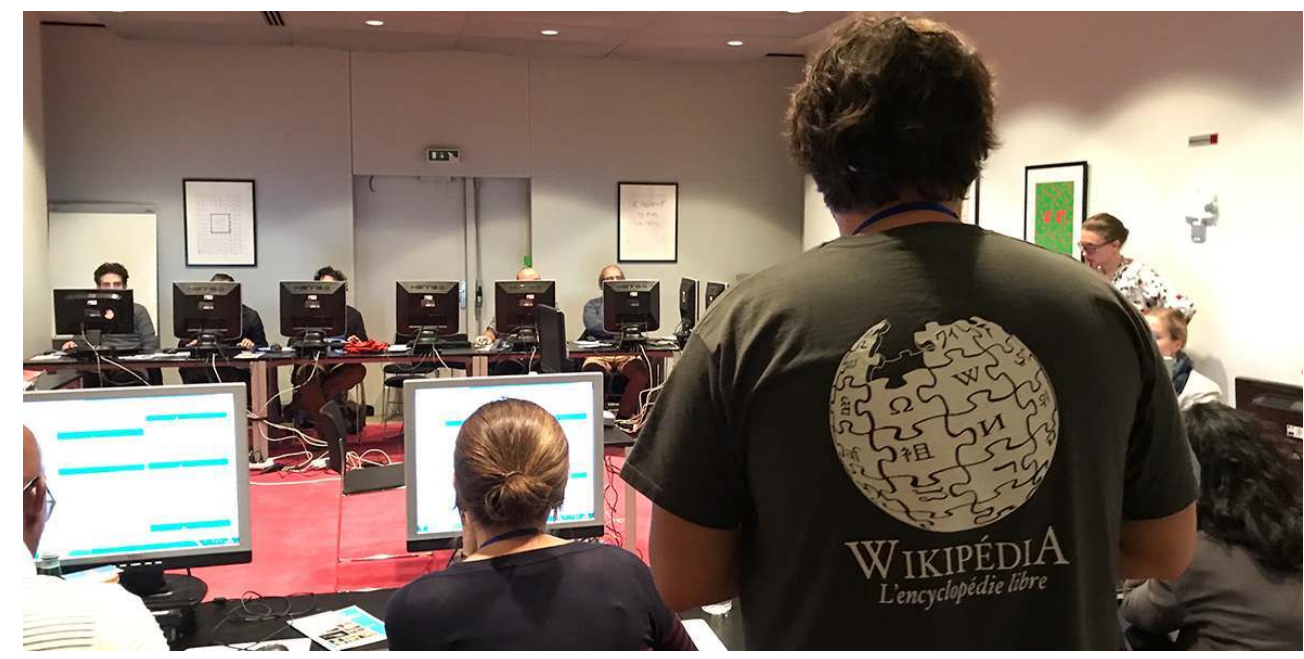

Edit-a-ton dédié au PCI organisé par le DPRPS et I'InOc Aquitaine en collaboration avec Wikimédia France à l'occasion de la présentation publique de PCILab, au ministère de la Culture, le 3 octobre 2017.

(c) Jean-Jacques Castéret, octobre 2017.

\section{Conclusion}

35 À la croisée de la recherche et de l'action, entre inventaire et éducation artistique et culturelle, appropriation des ressources par les artistes et acteurs de terrain et appropriation des fonctions collaboratives de ces médias par les individus et les communautés, le numérique occupe, dans la chaîne patrimoniale, une position médiane. Centre de ressources et courroie d'entraînement dans le cadre de médiations et d'animations de réseaux, ce simple élément de la chaîne est pourtant aujourd'hui stratégique dans le contexte mondial où tend à dominer la culture numérique ${ }^{33}$. Certes, il ne remplace pas les pratiques mais il les nourrit, les stimule. Vecteur de la construction des représentations, il en devient ainsi, à bien des égards, une part constitutive. Ces jalons d'une histoire en marche, pour partie encore expérimentale dans le champ du PCI, définissent ainsi le média numérique autant comme un outil de diffusion que comme l'organe au travers duquel se dit, se (re)constitue, la communauté : celle des groupes de pratiques, dans le cas des sites internet et plus spécifiquement des réseaux sociaux, ou celle des acteurs du PCI, dans le cas de PciLab. En l'espèce, le numérique tend à devenir un «lieu» du dialogue entre l'État ou les collectivités territoriales et les associations, les acteurs culturels et les chercheurs, le lieu d'une "sauvegarde immatérielle " $^{34}$, dans le sens d'un service public pour le PCI et la diversité culturelle. 


\section{NOTES}

1. - HOTTIN, Christian. «Sept ans, l'âge de raison. Dynamiques et enjeux du patrimoine culturel immatériel ». Dans KHAZNADAR, Chérif. Internationale de l'imaginaire. n.s., $\mathrm{n}^{\circ} 25$, Le patrimoine culturel immatériel. Premières expériences en France, 2011, p. 41-43.

2. - GRENET, Sylvie. «Les inventaires en France». Dans KHAZNADAR, Chérif. Op. cit., p. 77-91; QUIMBERT, Charles. "Le Patrimoine culturel immatériel, un enjeu de société. Autour d'une démarche en cours de réalisation en Bretagne ». Dans KHAZNADAR, Chérif. Op. cit., p. 93-106.

3. - Nous nous situons ici dans le contexte français où le DPRPS du ministère de la Culture est pleinement engagé au côté des chercheurs et des communautés de pratiques. En effet, PCI et numérique se conjuguent aussi dans d'autres pays sans pour autant que l'État ou les collectivités participent de la démarche. Voir CACHAT, Séverine et SEVERO, Marta. Patrimoine culturel immatériel et numérique. Paris : L'Harmattan, coll. « Humanités numériques », 2017.

4. - Voir DALBÉRA, Jean-Pierre et PASCON, Jean-Louis. Patrimoine culturel et multimédia. Paris : Mission de la recherche et de la technologie, Département de l'organisation et des systèmes d'information, Direction de l'administration générale, ministère de la Culture et de la Communication, 1999, p. 13.

5. - Voir COLLIN, Jean-François (coord.). Évaluation de la politique publique de numérisation des ressources culturelles. Paris : Services du Premier ministre, 2015, p. 8.

6. - Seules les collectes de Pierre Boissière font alors l'objet d'une numérisation financée par le conseil général du Lot-et-Garonne et mise en œuvre par le Conservatoire occitan (Toulouse-MidiPyrénées) qui réalise à cette occasion la description du fonds.

7. - Le fonds Junqué d'Oc, dont la numérisation puis la documentation ont été financées dans les premières années de la BnsA.

8. - Voir le site : http://www.sondaqui.com/ [consulté le 27/09/2017].

9. - Voir le site: http://aquitaine.culture.gouv.fr/dossiers-thematiques/langues-patrimoineimmateriel/occitan/bfcae23830d3ac58b5fdc084d925958c/ [consulté le 27/09/2017].

10. - Voir le site : http://jocondelab.iri-research.org/jocondelab [consulté le 27/09/2017].

11. - JocondeLab a été créé par l'Institut de recherche et d'innovation du Centre GeorgesPompidou et le ministère de la Culture et de la Communication. Pour plus de détails, voir le site : http://jocondelab.iri-research.org/jocondelab/about/ [consulté le 27/09/2017].

12. - Voir Pci-lab.fr, le site de l'Inventaire français du patrimoine culturel immatériel : https:// www.pci-lab.fr/ [consulté le 31/10/2017.

13. - Voir CASTÉRET, Jean-Jacques et LARCHÉ, Mélanie. « Le projet "PCI Lab" pour la valorisation numérique de l'Inventaire français du PCI ». Dans CACHAT, Séverine et SEVERO, Marta. Op. cit., p. $147-162$.

14. - CASTÉRET, Jean-Jacques et HEINIGER-CASTÉRET, Patricia. «Un exemple de PCI des communautés culturelles françaises : L'Inventaire pilote du PCI d'expression occitane en Région Aquitaine ». Dans LANKARANI, Leila et FINES, Francette (dir.). Patrimoine culturel immatériel et collectivités infraétatiques. Dimensions juridiques et régulation. Paris : Pedone, 2013, p. 41-54.

15. - Trouver, grâce aux liens hypertexte, des informations inattendues mais intéressantes, bien qu'elles soient hors du sujet initial.

16. - Voir le site: http://www.sondaqui.com/Patrimoine-vivant/le-pci-un-patrimone-vivant [consulté le 27/09/2017].

17. - Voir le site : http://www.sondaqui.com/Fetes-patronales/laruns [consulté le 27/09/2017]. 
18. - Ils suivent la classification des fiches d'inventaire accessibles sur le site du ministère. Voir le site: http://www.culturecommunication.gouv.fr/Politiques-ministerielles/Patrimoine-culturelimmateriel/Inventaire-en-France/Inventaire/Fiches-de-l-inventaire-du-patrimoine-culturelimmateriel.

19. - Voir le site : http://www.sondaqui.com/Noel/noel-feux [consulté le 27/09/2017].

20. - Voir le site : http://www.sondaqui.com/catalogue-regional [consulté le 27/09/2017].

21. - Les restrictions à la consultation des fonds produits dans le cadre des grandes collectes institutionnelles de ce musée laboratoire participent d'une logique de mise à distance des "profanes ", à commencer par les praticiens des musiques populaires - elles sont d'ailleurs reçues comme une confiscation -, mais aussi, de fait, de l'ensemble des chercheurs. Voir à ce propos: FABRE, Daniel, GASNAULT, François et LE GONIDEC, Marie-Barbara. Sources, Archives et Histoire Institutionnelle de l'Ethnomusicologie de la France, Programme de recherches du Laboratoire d'anthropologie et d'histoire de l'institution de la culture de l'Institut interdisciplinaire d'anthropologie du contemporain - CNRS / MCC / EHESS, 2015-2016. CHARLES-DOMINIQUE, Luc. "Sauvegarder aujourd'hui les musiques de tradition orale en France ». Dans SOLOMOS, Makis, CAULLIER, Joëlle, CHOUVEL, Jean-Marc, OLIVE, Jean-Paul. Musique et globalisation: une approche critique, actes du colloque "Musique et globalisation». Paris: Sampzon, Delatour, 2012, p. $165-176$.

22. - Loi $\mathrm{n}^{\circ} 2013-595 \mathrm{du} 8$ juillet 2013 d'orientation et de programmation pour la refondation de l'école de la République ; circulaire du 3 mai 2013 commune aux deux ministres et instituant la notion de " parcours d'éducation artistique et culturelle ».

23. - Voir le site : https://www.youtube.com/watch?v=usjOFHmzpAk.

24. - Sur ces aspects voir aussi SIBERS, Jean-François. "Problématiques patrimoniales, efflorescences territoriales ". Patrimoine. Enjeux contemporains de la recherche. Culture et recherche, $\mathrm{n}$ ○ 133, été 2016, coordonné par LIÉVAUX, Pascal et HOTTIN, Christian, p. 63-64.

25. - Voir le site : http://daquidoc.org/index.php?lang=fr. Il fait l'objet d'un accès réservé aux classes pilotes durant l'année scolaire 2016-2017. Son accès au grand public est prévu pour la rentrée scolaire 2017-2018.

26. - Voir le site : http://www.trobar-aquitaine.org/fr/ [consulté le 27/09/2017].

27. - Voir le site : http://www.in-oc.org/fr/ [consulté le 27/09/2017].

28. - Voir le site : http://www.patrimoine-immateriel-aquitaine.org [consulté le 27/09/2017].

29. - Voir le site : https://pci.hypotheses.org/1084 [consulté le 27/09/2017].

30. - Voir le site: http://item.univ-pau.fr/live/20-Programmes+de+recherche/027-Patrimoine +culturel+immat\%C3\%A9riel [consulté le 27/09/2017].

31. - Voir le site : http://pci.hypotheses.org/ [consulté le 27/09/2017].

32. - Wikidata est une base de données libre, collaborative et multilingue. Elle collecte les données utilisées par l'ensemble des productions de la fondation Wikimédia (Wikipédia, Wikimedia Commons...) et, au-delà, d'autres données structurées permettant d'alimenter l'ensemble de ces productions. Voir le site: https://www.wikidata.org/wiki/ Wikidata:Introduction/fr [consulté le 27/09/2017].

33. - DOUEIHI, Milad. La Grande conversion numérique. Paris : Éd. du Seuil, 2008.

34. - Voir HEINICH, Nathalie. "Synthèse et débats: un inventaire pour l'Europe?». Dans Le Patrimoine culturel immatériel de l'Europe: inventer son inventaire. Journée d'étude du 30 novembre 2007, Institut national du Patrimoine. "Comment administrer, inventorier, fixer, reproduire, décrire, etc., ce qui est de l'ordre de la pratique mouvante, du processus, de la représentation contextualisée? À cette question finale, je serais tentée d'apporter une réponse lapidaire: à patrimoine immatériel, sauvegarde immatérielle. En d'autres termes : l'enquête ethnologique et le travail d'inventaire ne sont-ils pas, logiquement, le seul mode de conservation réellement praticable du patrimoine culturel immatériel?» 


\section{RÉSUMÉS}

La diffusion, en France, de la notion de Patrimoine Culturel Immatériel a suivi deux voies simultanées. Un mouvement «top-down» de mise en œuvre de la Convention de l'Unesco (2003) par le ministère de la Culture à travers la création d'un inventaire et par la mobilisation des dispositifs jusqu'alors dédiés au patrimoine ethnologique. Envisagée comme un levier par les communautés de praticiens, la Convention a également généré un mouvement «bottom-up » : candidatures sur les listes de l'Unesco, participation aux inventaires. Chacun d'eux a permis l'instauration d'un dialogue renouvelé entre l'Etat, les collectivités, les associations ou acteurs de tous ordres. Les questions de transmission et de sauvegarde (donc d'action) étant centrales dans la Convention, quel est donc aujourd'hui la nature de ce dialogue? Quels en sont les cadres ? Pour quels résultats naissants? A la croisée de ces deux mouvements et à partir de l'expérience conduite par l'ethnopôle InOc Aquitaine, partagée avec le Département du pilotage de la recherche et de la politique scientifique (DPRPS) du ministère et divers acteurs du PCI du territoire français, cette contribution s'attache plus particulièrement aux stratégies numériques déployées, et notamment à la place des dispositifs produits dans la chaîne patrimoniale à la croisée de la recherche et de l'action; entre inventaire et éducation artistique et culturelle; appropriation des ressources par les artistes et acteurs de terrain, et appropriation des fonctions collaboratives de ces médias par les individus et les communautés.

In France, the diffusion of the concept of intangible cultural heritage followed two simultaneous paths. A 'top-down' movement for the implementation of the Unesco Convention (2003) by the French Ministry of Culture, by means of the creation of an inventory and the mobilisation of the techniques hitherto used for the ethnological heritage. Considered as a lever by communities of practitioners, the convention has also generated a 'bottom-up' movement: nominations for the Unesco lists, participation in inventories... Each of these movements has allowed for the establishment of a renewed dialogue between the State, communities and associations and actors of all kinds. Since the issues of transmission and safeguarding (and therefore action) are central to the Convention, what is the nature of this dialogue today? What are its frameworks? For what emerging results? At the crossroads of these two movements and on the basis of the experience led by the Inoc Aquitaine ethnopole, shared with the Département du pilotage de la recherche et de la politique scientifique (DPRPS) at the Ministry of Culture and various ICH actors in France, this contribution focuses on the digital strategies deployed and at the place of the devices produced in the heritage chain at the crossroads of research and action; between inventory and arts and cultural education; appropriation of resources by artists and field actors, and appropriation of the collaborative functions of these media by individuals and communities. 


\section{INDEX}

Mots-clés : patrimoine culturel immatériel, numérique, inventaire du patrimoine culturel, médiation, éducation artistique et culturelle, occitan, langues de France, Nouvelle-Aquitaine, ethnopôle, Aquitaine cultures connectées, archive orale, Wikipédia, collaborativité

Keywords : intangible cultural heritage, digital, inventorisation, mediation, artistic and cultural education, occitan, minority languages, Nouvelle-Aquitaine, ethnopôle, Aquitaine cultures connectées, oral archives, Wikipedia, collaboration

\section{AUTEUR}

\section{JEAN-JACQUES CASTÉRET}

Ethnopôle InOc Aquitaine / Laboratoire ITEM de l'Université de Pau et des Pays de l'Adour jj.casteret@in-oc.org 